\title{
CAPACITIVE TOMOGRAPHY FOR THE LOCATION OF PLASTIC PIPE
}

\author{
QUARTERLY TECHNICAL REPORT
}

(April 1 through June 30, 2003)

\author{
Principal Investigator: Brian J. Huber \\ Project Manager: Christopher J. Ziolkowski \\ 847-768-0549, chris.ziolkowski@gastechnology.org
}

$\underline{\text { Report Issue Date: July 29, } 2003}$

DOE Contract \#: DE-FC26-01NT41161

Submitted by

Gas Technology Institute 1700 South Mount Prospect Road

Des Plaines, Illinois 60018

GTI Project Number: 61140

\author{
Submitted to \\ NETL AAD Document Control Bldg. 921 \\ U.S. Department of Energy \\ National Energy Technology Laboratory \\ P.O. Box 10940 \\ Pittsburgh, PA 15236-0940
}

DOE Project Officer

Anthony Zammerilli 


\section{DISCLAIMER}

"This report was prepared as an account of work sponsored by an agency of the United States Government and the Gas Technology Institute (GTI). Neither the United States Government, nor GTI, nor any agency thereof, nor any of their employees, makes any warranty, express or implied, or assumes any legal liability or responsibility for the accuracy, completeness, or usefulness of any information, apparatus, product, or process disclosed, or represents that its use would not infringe privately owned rights. Reference herein to any specific commercial product, process, or service by trade name, trademark, manufacturer, or otherwise does not necessarily constitute or imply its endorsement, recommendation, or favoring by the United States Government, GTI, or any agency thereof. The views and opinions of authors expressed herein do not necessarily state or reflect those of the United States Government or any agency thereof." 


\begin{abstract}
Throughout the utility industry, there is high interest in subsurface imaging of plastic, ceramic, and metallic objects because of the cost, reliability, and safety benefits available in avoiding impacts with the existing infrastructure and in reducing inappropriate excavations. Industry interest in locating plastic pipe has resulted in funding available for the development of technologies that enable this imaging. Gas Technology Institute (GTI) proposes to develop a compact and inexpensive capacitive tomography imaging sensor that takes the form of a flat plate or flexible mat that can be placed on the ground to image objects embedded in the soil.
\end{abstract}

A compact, low-cost sensor that can image objects through soil could be applied to multiple operations and will produce a number of cost savings for the gas industry. In a stand-alone mode, it could be used to survey an area prior to excavation. The technology would improve the accuracy and reliability of any operation that involves excavation by locating or avoiding buried objects. An accurate subsurface image of an area will enable less costly keyhole excavations and other cost-saving techniques.

Ground penetrating radar (GPR) has been applied to this area with limited success. Radar requires a high-frequency carrier to be injected into the soil: the higher the frequency, the greater the image resolution. Unfortunately, high-frequency radio waves are more readily absorbed by soil. Also, highfrequency operation raises the cost of the associated electronics. By contrast, the capacitive tomography sensor uses low frequencies with a multiple-element antenna to obtain good resolution. Low-frequency operation lowers the cost of the associated electronics while improving depth of penetration.

The objective of this project is to combine several existing techniques in the area of capacitive sensing to quickly produce a demonstrable prototype. The sensor itself will take the form of a flat array of electrodes that can be inexpensively fabricated using printed circuit board techniques. The image resolution is proportional to the number and spacing of the electrodes in the array. Measuring the complex impedance between adjacent electrodes at multiple frequencies forms the image. Simple location of plastic pipe with a two-electrode array has already been demonstrated.

Thus far, 4-element and 16-element sensor arrays have been fabricated on 24" by 24" printed circuit boards and tested. The sensor arrays have been tested with buried plastic piping at GTI both in soil boxes and an outdoor facility. Sensitivity to the presence of plastic pipe in soil has been demonstrated with 2", 4", and 6" diameter pipes at depths greater than 4 feet. This sensitivity is unaffected by soil moisture conditions. A 64-element array is currently being fabricated to provide greater spatial resolution of buried objects. 


\section{TABLE OF CONTENTS}

\section{$\underline{\text { Page }}$}

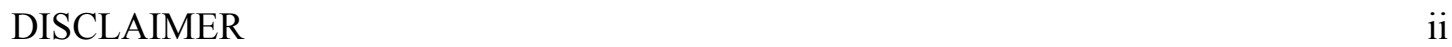

$\begin{array}{lll}\text { ABSTRACT } & \text { iii }\end{array}$

EXECUTIVE SUMMARY 1

$\begin{array}{ll}\text { INTRODUCTION } & 2\end{array}$

EXPERIMENTAL 3

EXPERIMENTAL RESULTS 3

$\begin{array}{ll}\text { IMAGING THE SENSOR DATA } & 6\end{array}$

SOFTWARE DEVELOPMENT 9

$\begin{array}{lr}\text { MATLAB ANALYSES } & 10\end{array}$

SUPPORT ELECTRONICS 13

$\begin{array}{ll}\text { SENSOR DEVELOPMENT } & 15\end{array}$

$\begin{array}{ll}\text { CONCLUSIONS } & 19\end{array}$

WORK PERFORMED THIS QUARTER 20

REFERENCES 22

LIST OF ACRONYMS AND ABBREVIATIONS 23

APPENDIX A - MATLAB CODE FOR SENSOR ANALYSIS 24 


\section{LIST OF GRAPHICAL MATERIALS}

$\underline{\text { Page }}$

Fig. 1. Scan of 6" PE Pipe at 54" Depth in Outdoor Facility 3

Fig. 2. Scan of 4" PE Pipe at 42" Depth Indoor Soil Pit 4

Fig. 3. Scan of Indoor Soil Pit for 8 Elements 5

Fig. 4. Pipe Beneath 1st Row of Sense Elements $\quad 6$

Fig. 5. Pipe Beneath $2^{\text {nd }}$ Row of Sense Elements $\quad 7$

Fig. 6. Pipe Angled Beneath Corner of Array $\quad 7$

Fig. 7. Pipe Angled Diagonally Beneath Array $\quad 8$

Fig. 8. Test of 64-Element GUI 9

Fig. 9. Signal Variation versus Drive Impedance for a 5 pF Sense Element 11

Fig. 10. Sensor Field Simulation With Pipe in Near Field 12

Fig. 11. Sensor Field Simulation Pipe in Far Field 12

Fig. 12. Block diagram of the Multiplexer and Buffer board 13

Fig. 13. Multiplexer and buffering board 14

Fig. 14. 64-Element Sensor Board Layout $\quad 15$

Fig. 15. Sensor Side of Completed 64-Element Board 16

Fig. 16. Close Up of Component Side Showing Multiplexer and Amplifiers 16

Fig. 17. Cart with 16-Element Sensor array and Electronics 17

Fig. 18. Bottom of Cart Showing Array 18

$\begin{array}{lr}\text { Fig. 19. Cart and Operator } & 18\end{array}$ 


\section{EXECUTIVE SUMMARY}

Excavation is an inherently expensive and risky operation that utilities seek to minimize. The cost of an excavation can range from $\$ 500$ to $\$ 5,000$ depending on the size and location. Extremely small (or, keyhole) excavations require very accurate targeting to be effective. Full-size excavations need to span the desired subsurface features in the first attempt, and rework of any type of excavation is expensive and disruptive. Less easily quantified are the losses incurred during dig-ins or impacts with the existing buried infrastructure resulting from faulty location data. These incidents require the mobilization of whatever resources are required to effect immediate repair, disrupting other operations. A release of gas, water, or a breach of an electrical main can severely affect the safety of workers and the public.

Directional boring is another technology that is being used to reduce the number of excavations. In this operation, a boring tool is used to create a pilot tunnel between two widely spaced pits. The boring tool is then pulled back to the entry pit drawing new plastic pipe with it. There have been instances of plastic pipe inadvertently intersecting clay sewer lines during the directional boring operation. When attempts were made to clear the blocked sewer line, the plastic gas pipe was breached, filling the sewer system with gas. There is at least one documented instance of an explosion caused by this situation. This hazardous situation could have been prevented with better subsurface imaging. Specifically, there is a need to accurately image non-metallic sewer lines as well as the plastic pipe.

In light of the consequences of faulty location data, the gas industry would be quick to adopt a subsurface imaging technology that meets their criteria. GTI industry advisors have identified this area as a high priority as demonstrated by the efforts expended on it to date. Current subsurface technologies to image non-metallic pipes, such as GPR and acoustic locators, are in limited use. A better technology would have excellent prospects for commercial deployment.

Because of its access to gas industry research, GTI is familiar with the merits and shortcomings of the various methods of subsurface imaging that have been attempted. The proposed capacitive tomography-imaging sensor directly addresses several deficiencies of the currently available technologies. The capacitive tomography technique is sensitive to the presence of plastic and ceramic piping materials. In addition, the thin-film nature of the sensor makes it adaptable to multiple applications. Also, capacitive tomography will give greater depth of penetration at a lower cost than ground-penetrating radar. 


\section{INTRODUCTION}

The objective in this project is for GTI to apply existing research to reliably image subsurface features to benefit the natural gas distribution infrastructure. This innovation enables a compact low-cost sensor to be developed to detect buried plastic pipe, a long-standing challenge for the natural gas industry. A compact, low-cost sensor that can image objects through soil could be applied to multiple operations and will produce a number of cost savings for the gas industry. In a stand-alone mode, it could be used to survey an area prior to excavation. The technology would improve the accuracy and reliability of any operation that involves excavation by locating or avoiding buried objects. An accurate subsurface image of an area will enable less costly keyhole excavations and other cost-saving techniques.

The proposed technique performs a low-frequency impedance measurement using a multiple-element antenna array. The impedance of the soil and inclusions is sensitive to the dielectric properties of the inclusions as well as their conductivities. Thus, the technique is sensitive to plastic and metallic objects both.

A device that can reliably image objects through soil is a high priority for the gas infrastructure industry as indicated by the considerable effort already expended to develop locating and imaging subsurface technologies. The increased use of plastic piping materials has complicated this effort, and much existing technology is applicable only to metallic materials. The technology of GPR only partially fulfills this objective. While sensitive to plastic materials, GPR use has been limited due issues with depth of penetration and expense.

An experimental capacitive scanning unit has successfully located plastic pipe in a GTIsupervised test during the mid-1990's. From the outset, this technique has demonstrated sensitivity to plastic pipe materials. An acoustic method and GPR were also tested during the same project. The test involved technology providers performing a "blind" location on pipes buried on GTI property. GTI personnel knew the location of the buried piping and the technology providers did not. The rudimentary capacitive tomography device that was tested demonstrated good sensitivity and was simpler than the other devices that were tested.

The current project effort by GTI has extended this early technology to provide a coarse image of buried objects. The original technique used 2 sense elements to locate the pipe. GTI has tested 4 and 16-element arrays to provide greater resolution of the pipe's location. These arrays can detect plastic pipe under more than 4' of soil with a high signal to noise ratio. A 64element array has been designed, fabricated, and is undergoing initial testing. 


\section{EXPERIMENTAL}

Experimental Results

Inclement spring weather prevented field-testing until early June of 2003 . When testing was started the soil was water saturated. Figure 3 shows a very strong signal for a 6 -inch plastic pipe buried at 4.5 feet of depth. The experimental apparatus consists of the sensor array mounted on a frame that slides on a set of tracks. In this experiment the array is moved relative to the buried pipe such that element 1,3,5, and 7 passes over the pipe in that sequential order. Elements 1,3, 5,and 7 are the prominent valleys from left to right. Each valley of the respective element indicates that the element is directly over the pipe.

data6_1_03_2

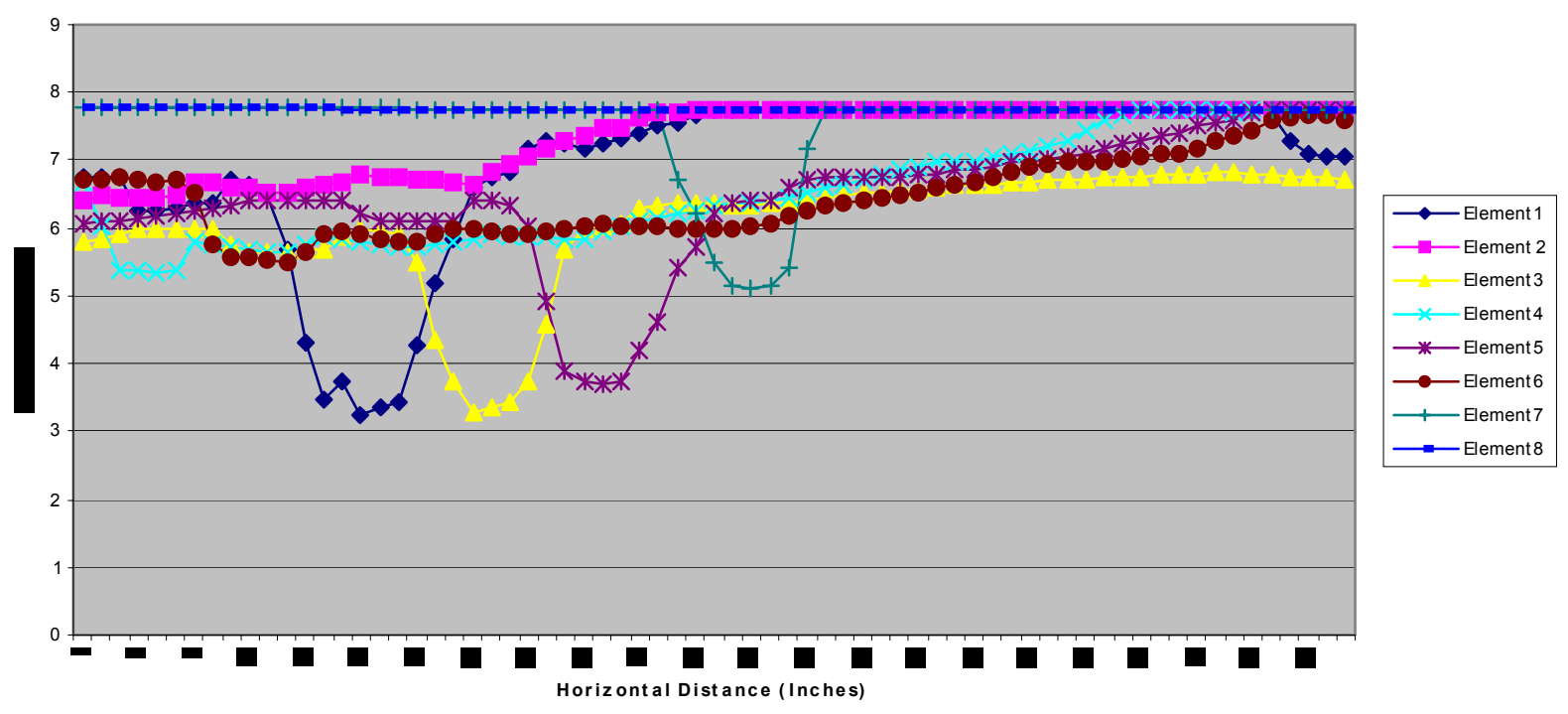

Fig. 1. Scan of 6" PE Pipe at 54" Depth in Outdoor Facility 
Pit_6_9_03_1.5V_100khz_9

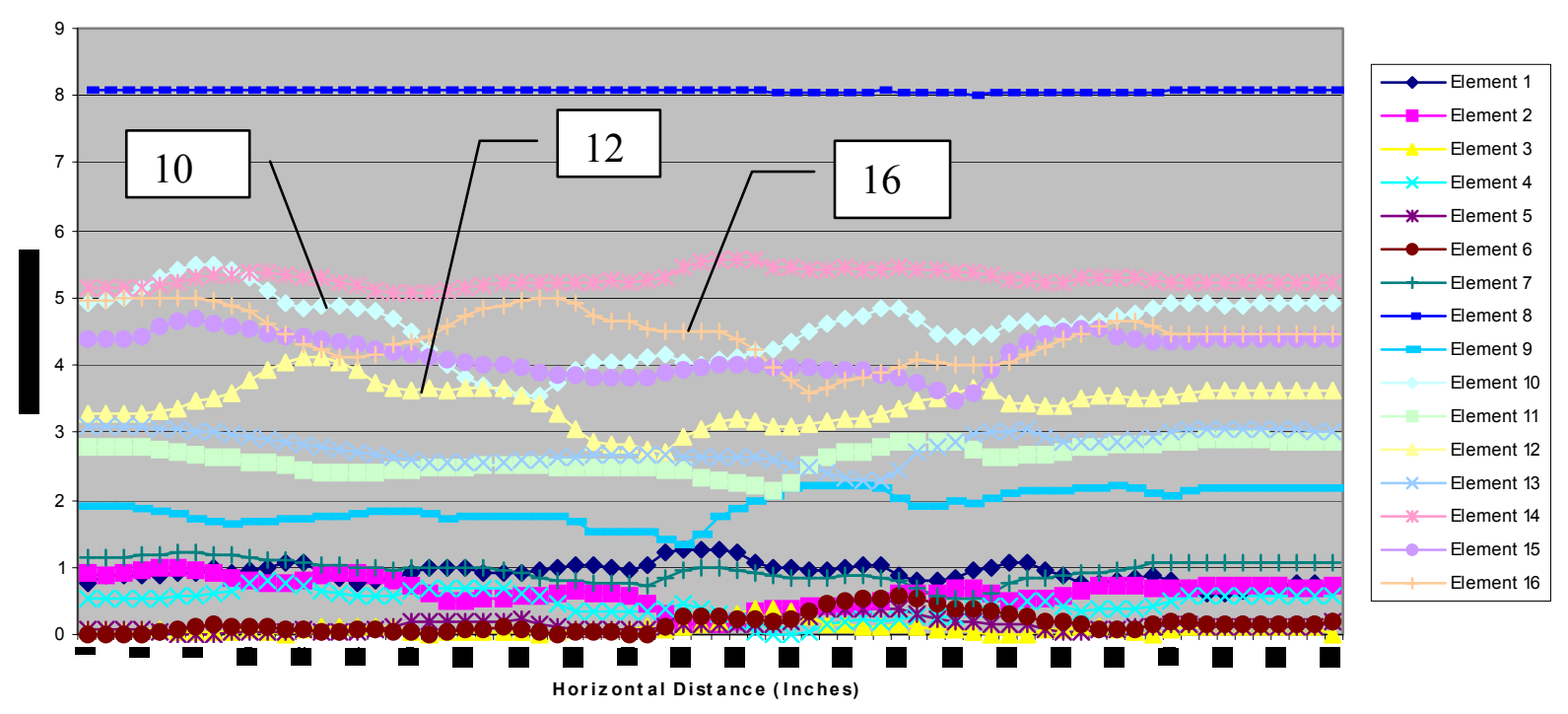

Fig. 2. Scan of 4" PE Pipe at 42” Depth Indoor Soil Pit

In Fig. 2 the array is being tested on an indoor soil test pit. The pit conditions were very dry and thus the signals are broader and less defined. The pipe in the soil pit is 4" diameter PE buried 42" deep. The array is moved past the stationary pipe such that various elements are directly over the pipe at different times. Elements 10, 12, and 16 clearly show the same perturbation of the signal as the respective array elements pass over the pipe location. 
Pit_6_9_03_1.5V_100khz_5

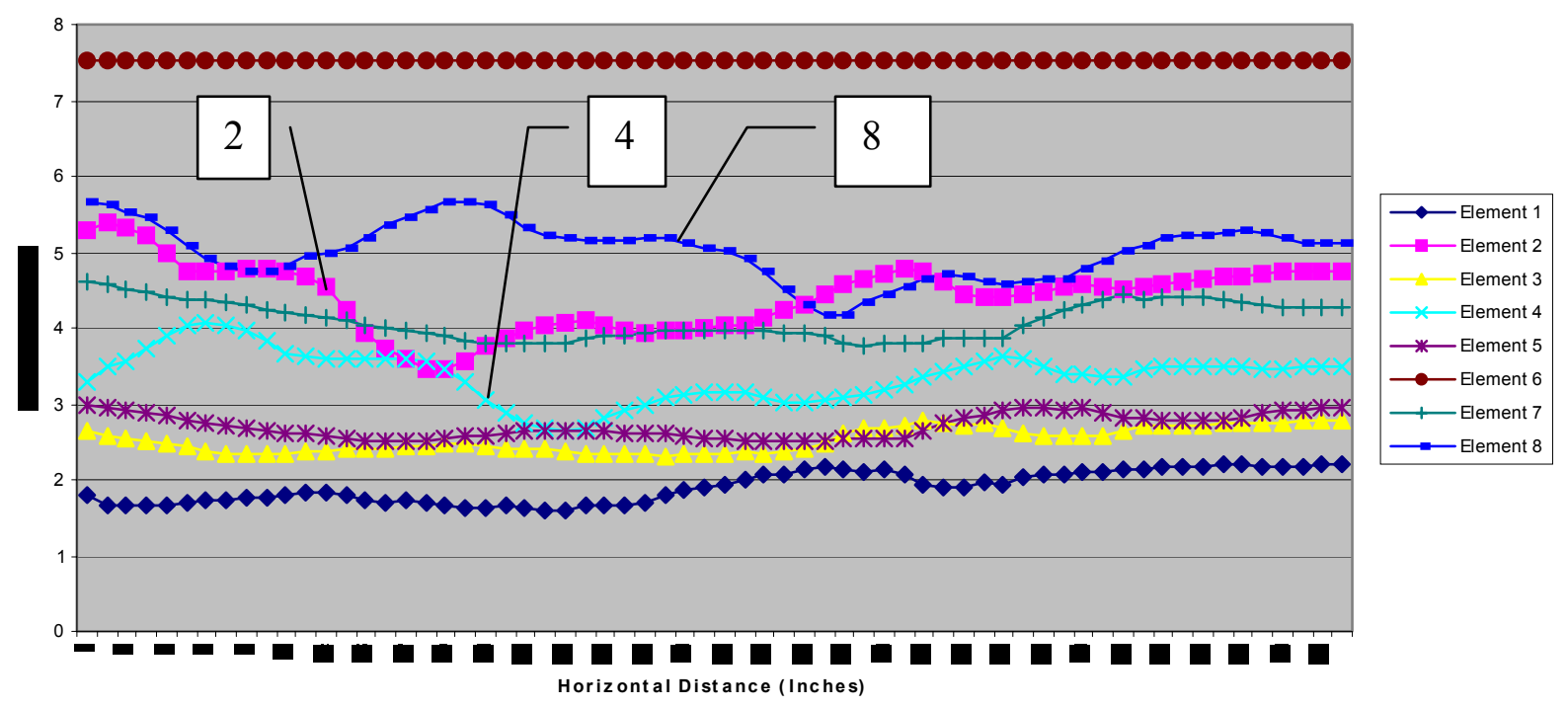

Fig. 3. Scan of Indoor Soil Pit for 8 Elements

In Figure 3 the same type pipe signature is in evidence as indicated by traces from element 2,4 and 8 . In order to see the signals more clearly only 8 of the 16 elements are scanned. It is also evident that not all the elements in the array show the same sensitivity to the presence of plastic.

The question arises why some of the elements seem to be very sensitive to the presence of the pipe while other elements have little or know sensitivity. There are several possible explanations for this. At times an element is simply experiencing a poor cable connection or broken solder joint. More often the variable drive capacitance is not adjusted for optimal sensitivity, thus while one element may produce a $500 \mathrm{mV}$ signal as it passes over the pipe another element may only produce a $50 \mathrm{mV}$ signal. When both signals are presented on the same graph the auto-scaling causes the smaller to appear flat. There is also variation in the size of the air gap between various sensor elements and the uneven ground surface. All of these variables can have a significant effect on the sensor and are difficult to remove. The "Sensor Development" section has some discussion of modifications to the hardware that are proposed to remedy some of these issues. 


\section{$\underline{\text { Imaging the Sensor Data }}$}

The following screens show representative imaging of the pipe. In this mode, the array is left stationary on the ground. The soil is scanned and the impedance differences between adjacent sense elements are mapped into colors. In Figure 4 elements 1,2,9, and 10 are being excited indicating the presence of the plastic pipe located directly under these elements running from left to right along the bottom edge of the array. In Figure 5, the array has been moved with respect to the pipe. Elements 3,4,11, and 12 are showing the location of the pipe directly below these elements.
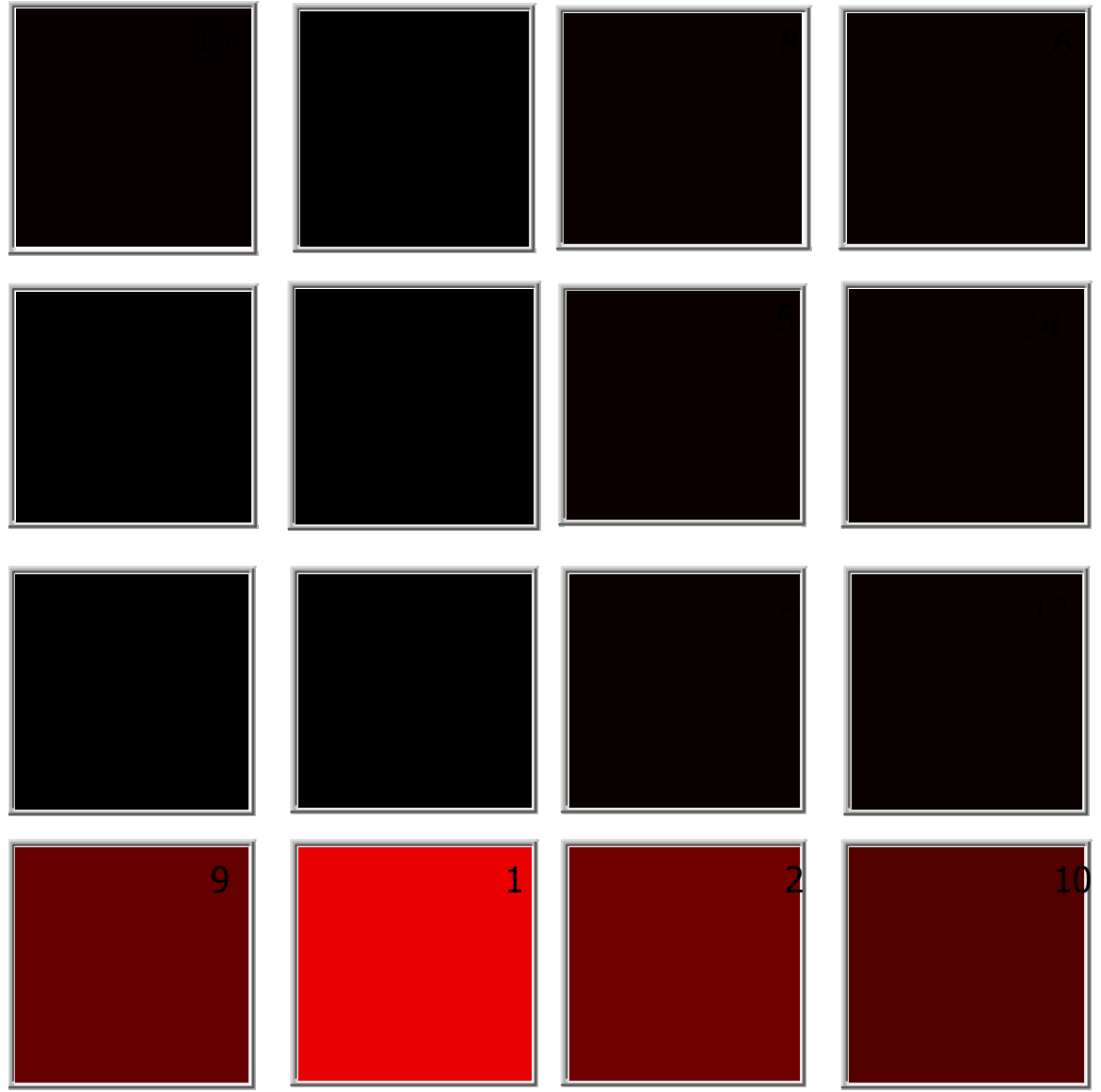

Fig. 4. Pipe Beneath 1st Row of Sense Elements 

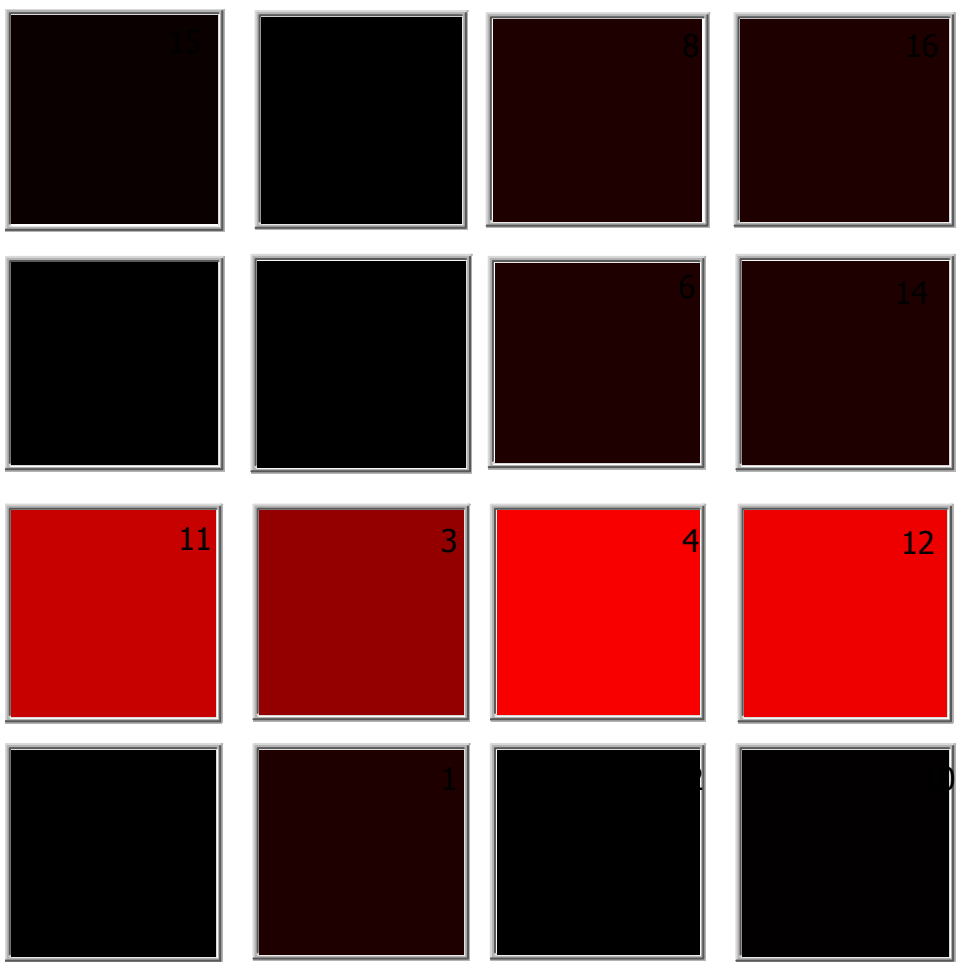

Fig. 5. Pipe Beneath $2^{\text {nd }}$ Row of Sense Elements
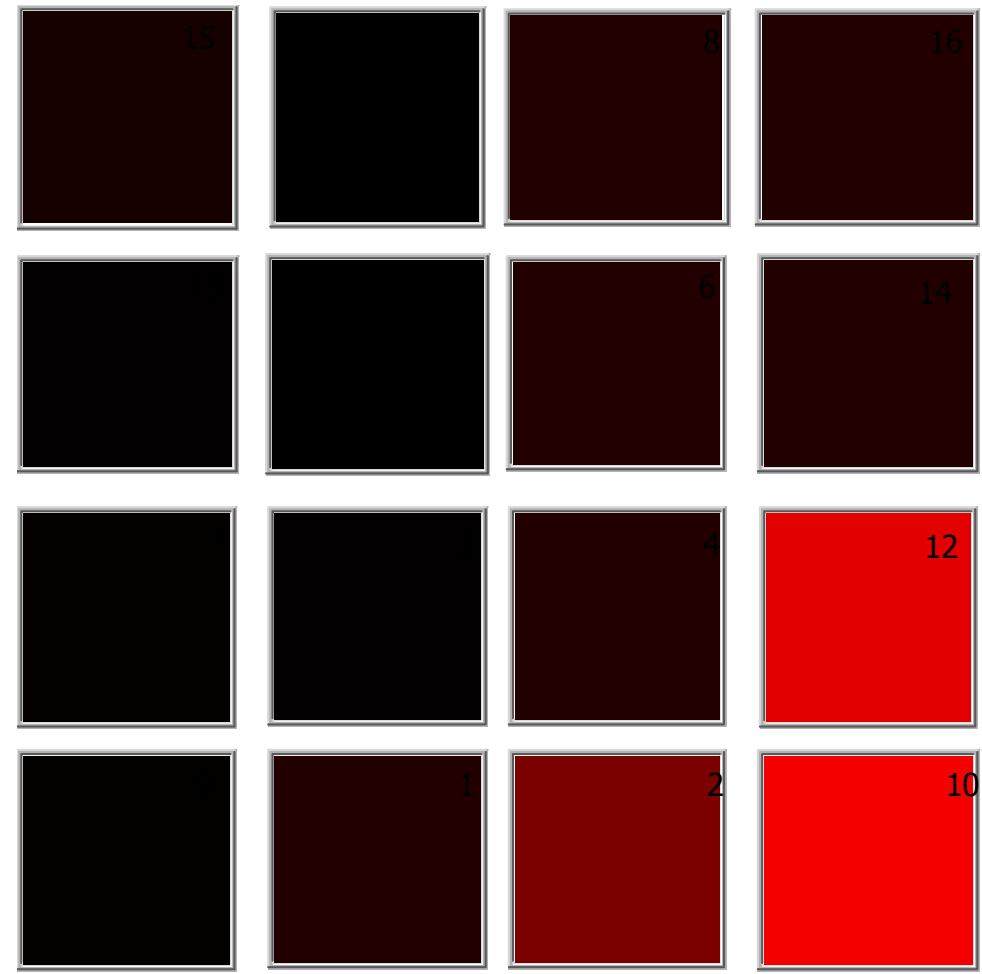

Fig. 6. Pipe Angled Beneath Corner of Array 
In Figure 6 elements 2,10, and 12 are excited indicating the pipe running at an angle from the lower left to the upper right under these elements. In Figure 7 elements 11, 5, and 8 are excited indicating a pipe running from the lower left to the upper right under the excited elements.
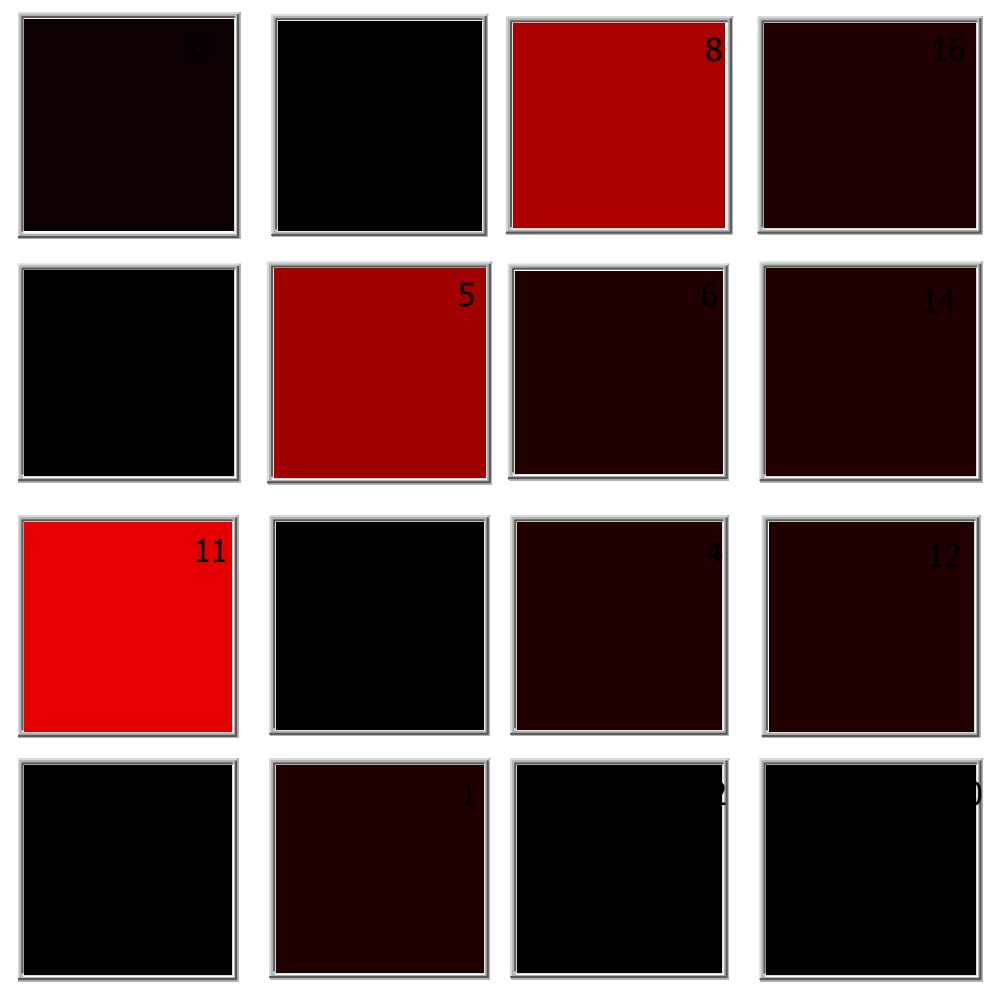

Fig. 7. Pipe Angled Diagonally Beneath Array 


\section{$\underline{\text { Software Development }}$}

New code has been developed in preparation for the 64-element sensor board. This code is very similar to previous code except it now is scanning 64 elements instead of 16 . There was concern before the 64-element board went out for fabrication that the speed of the PC would not scale up to accommodate the additional elements. The code was implemented and is now acquiring 64 elements. This code was tested by reading signals from the 16-element array 4 times, as shown in Figure 8. The display update speed did slow down by approximately 50\% but still updates the entire screen in less than a second. This does not significantly impair the usability of the GUI.

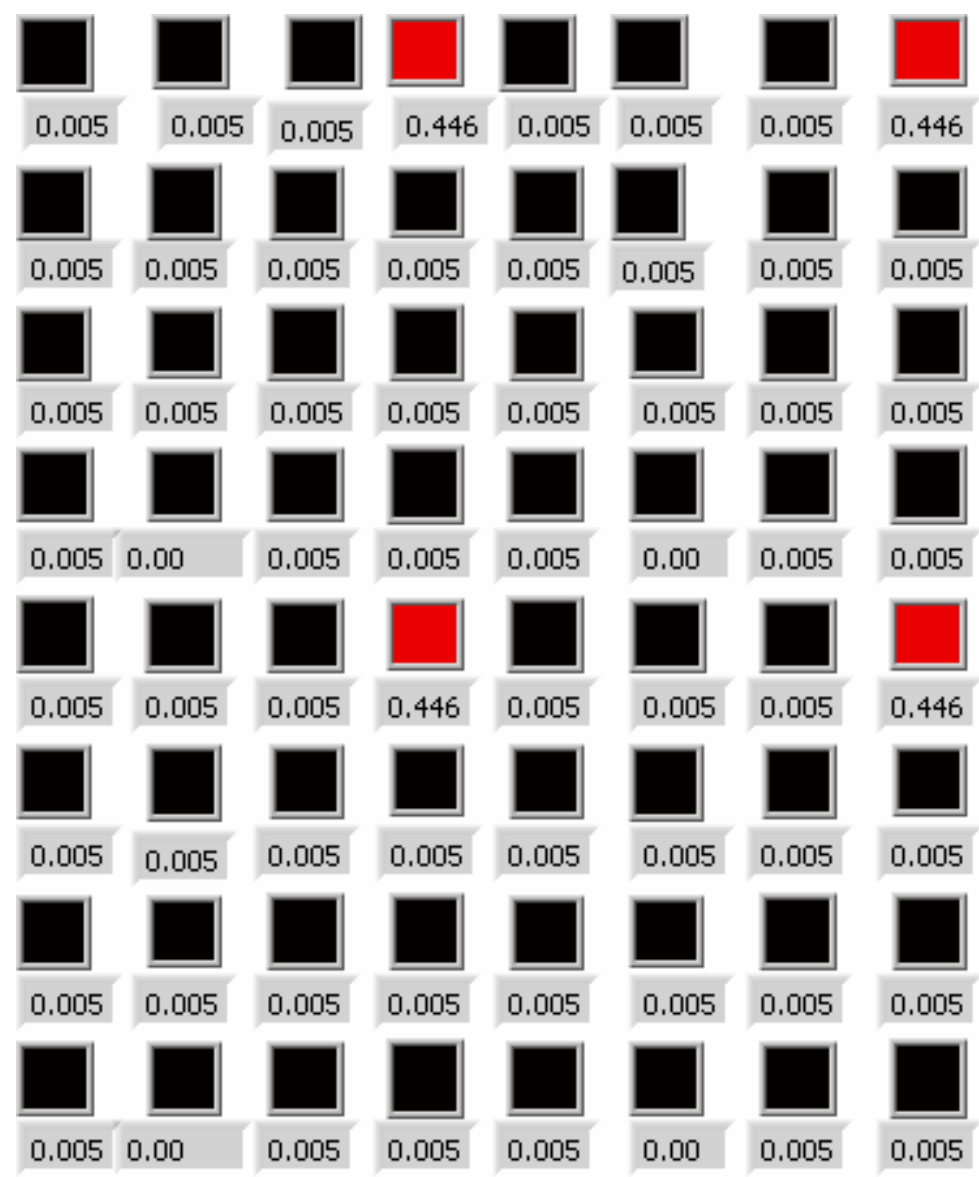

Fig. 8. Test of 64-Element GUI 


\section{MatLab Analysis of Sensor Sensitivity}

A numerical analysis was conducted to investigate the relative contribution to the sensitivity of the sensor arising from variations in the absolute magnitude of the sensor impedance vs. shifts in the phase angle of the sensor impedance. It has been the contention of the author that by sampling the sensor output waveform once per sample and averaging multiple samples a significantly larger signal can be achieved for a small change in sensor capacitance, than would be seen if one is simply measuring the RMS signal output. Experimental results support this premise.

This synchronous sampling is also known as synchronous demodulation or lock-in amplification. This method can significantly improve the signal to noise ratio of the feature of interest. It is necessary to have access to both the excitation (drive) signal and the sensor signal to achieve this synchronization. The exact phase at which the synchronous sample is obtained relative to the excitation signal is critical. The MatLab code in Appendix 1 calculates the change in the amplitude factor versus the change in phase factor for a small variation in sensor capacitance. Again this phase effect can only be seen when sampling once per cycle, and averaging together a large number of such samples.

The balance of the sensor elements also affects the sensitivity. Figure 9 confirms that the sensitivity of the sensor is at maximum when the impedance of the drive element and the sensor element are equal. Taking the derivative of the sensor voltage divider circuitry demonstrates this. Typical values of drive capacitance, sensor capacitance, and impedance were inserted into both sets of equations and plotted. The contribution to the overall variation from the amplitude factor vs. the phase factor was kept separate so that a comparison could be done. In other words a calculation was done whereby the output sensor voltage for one value of sensor capacitance was calculated and the then a new output sensor voltage for a slightly different value of sensor capacitance was computed.

When sampling once per cycle the output of a sensor element is made up of the product of an amplitude factor and a phase factor. These factors will change with a change of sensor capacitance. This calculation demonstrates the relative change of the amplitude factor vs. the phase factor for a small change in sensor capacitance. What was found was that the phase factor gave a factor of 10 larger contribution to the sensor output voltage than the amplitude factor for a small change in the sensor capacitance. Additionally that contribution from the phase factor is greatest when the sample is taken near the zero crossing. This is understandable given that a sine wave has its greatest slope at the zero crossing, and zero slope at its peak. 


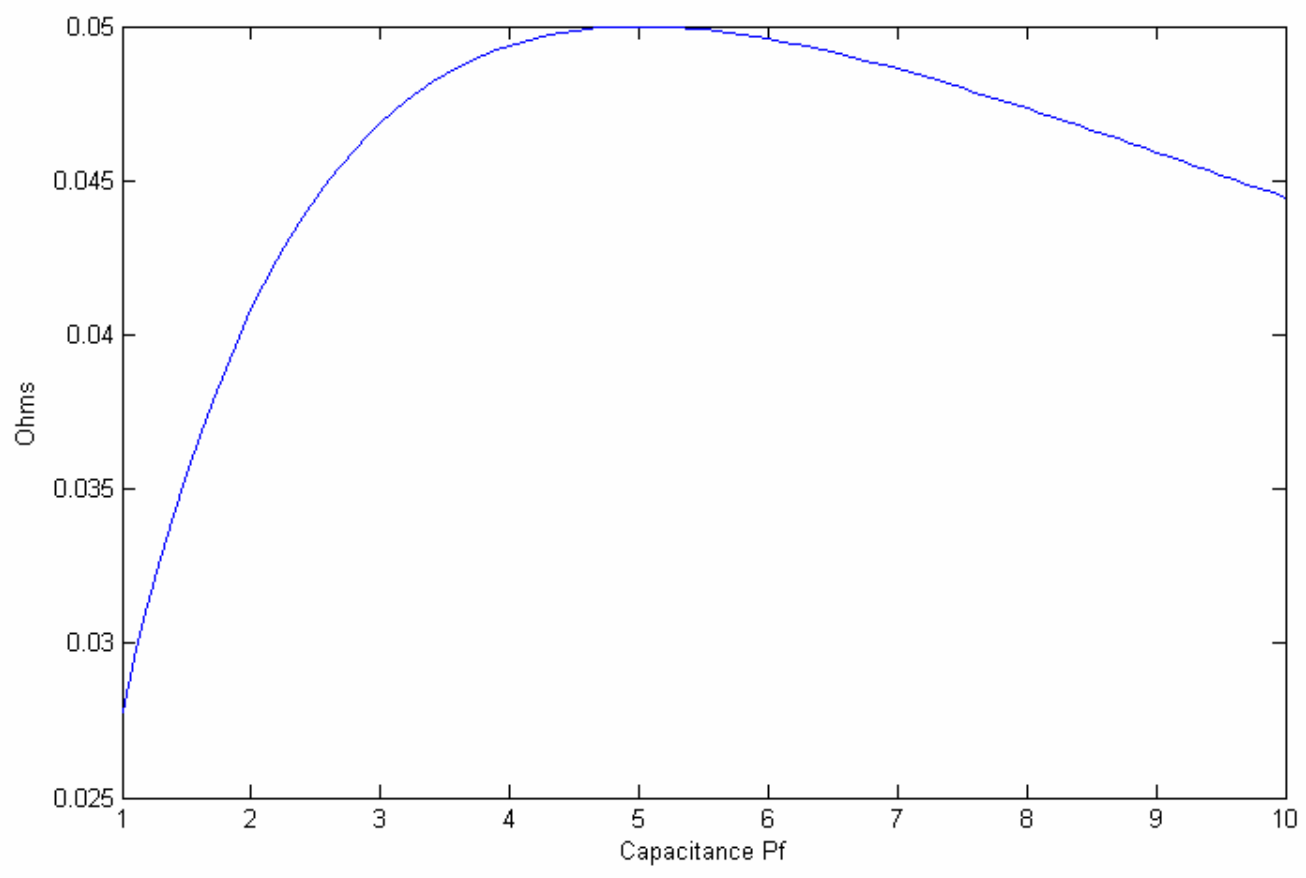

Fig. 9. Signal Variation versus Drive Impedance for a 5 pF Sense Element

\section{MatLab PDE ToolBox Simulation of Sensor Array}

A MatLab partial differential equation toolbox was used to assist in the design, understanding, and visualization of the electric field line distribution surrounding the sensor elements. This toolbox uses the finite element method to determine the solution of the partial differential equations that govern heat, sound, and electromagnetism. In particular the PDE to be solved for this application is LaPlace's equation $\nabla^{2} E=\rho \varepsilon_{0}$. The PDE toolbox allows a user to draw 2D geometries and then assign the boundary conditions. A mesh is generated and then a solution is found. Figures 10 and 11 show solutions for two simple pipe and sensor arrangements.

Arguably the PDE toolbox provides a more qualitative understanding, at least at first, than quantitative, but this tool does significantly motivate the conceptual probing of the problem. For instance the determination of appropriate boundary conditions suggests a more detailed analysis of the role that a static charge on the pipe may play. 


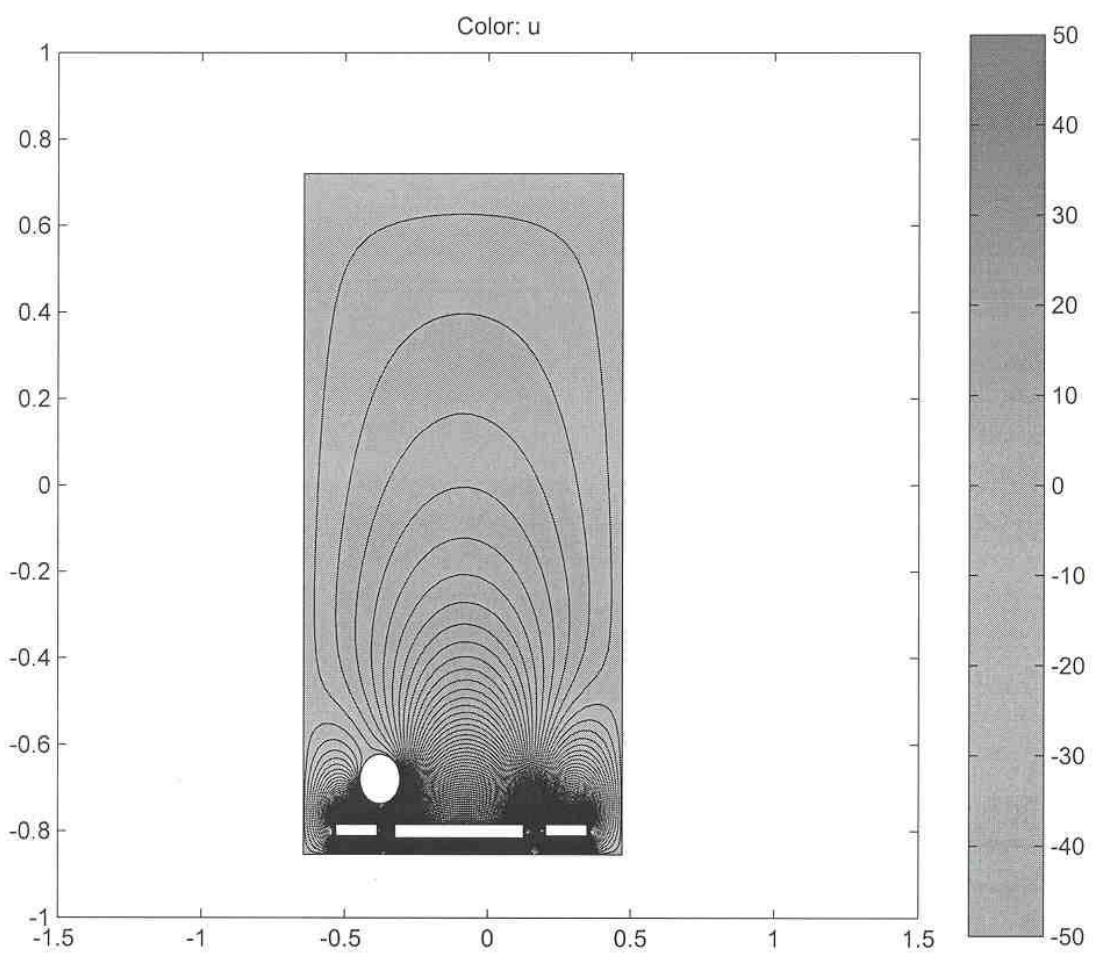

Fig. 10. Sensor Field Simulation With Pipe in Near Field

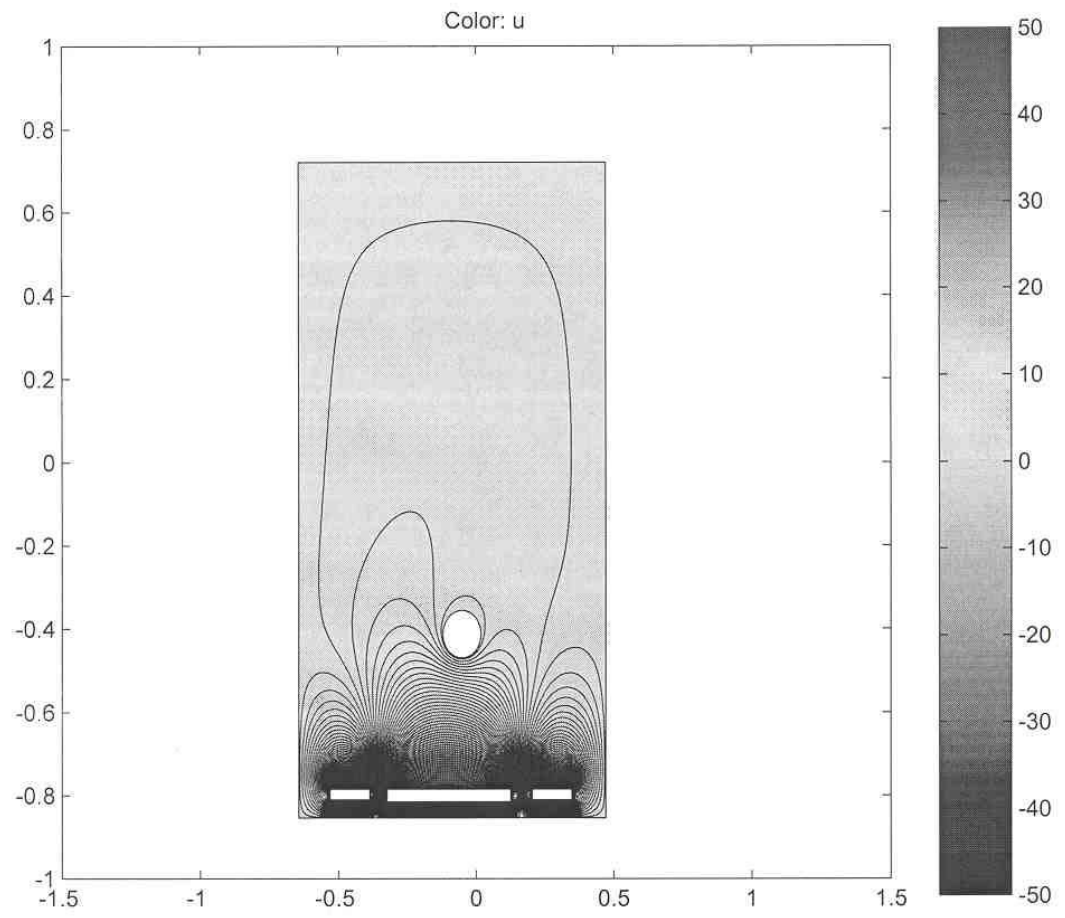

Fig. 11. Sensor Field Simulation Pipe in Far Field 


\section{$\underline{\text { Support Electronics }}$}

The sensor elements must be sequentially scanned in order to build up an image of what is beneath the sensor. Several items of support electronics are required to accomplish this. Amplification is required to raise the sensor signals to detectible levels and to "buffer" the signals from outside interferences. A multiplexer is required to select the sensor elements singly or in pairs. Also, appropriate connectors and cabling must be provided to route signals to the appropriate points.

The multiplexer and buffer circuit board was received in early January 2003. Integration and testing of this board moved forward. This board implements the multiplexing, buffering, and amplification for 16 analog input channels. Each channel is connected to a sense element on the sensor plate. Using the multiplexer to scan these elements sequentially allows an image to be built up. The solid-state bridge amplifier used to drive the sensor array was mounted in an aluminum box and power supply was designed, constructed and also mounted with the amplifier. Modularizing this critical piece of equipment allows it to be placed close to or adjacent to the sensor array.

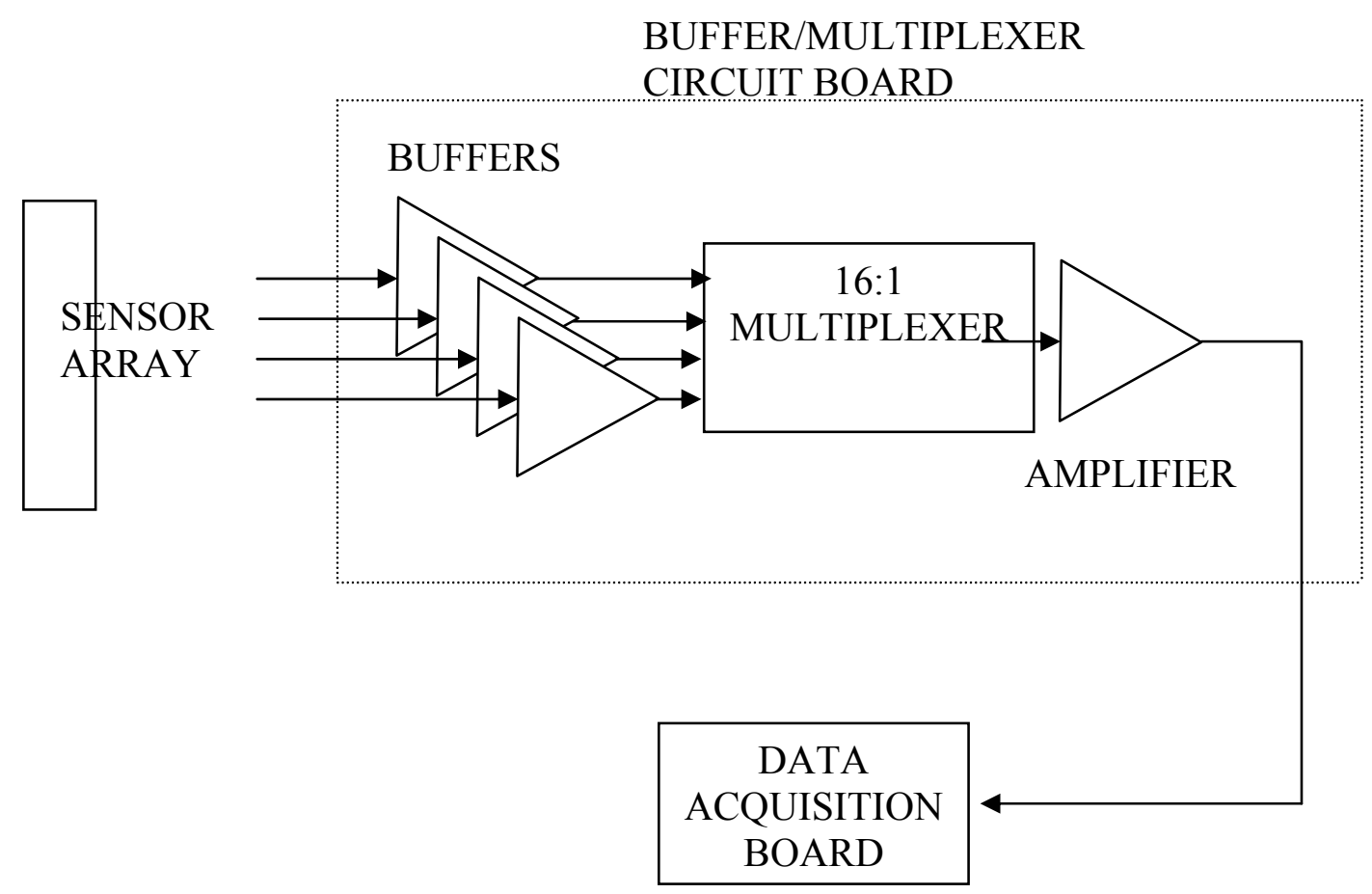

Fig. 12. Block diagram of the Multiplexer and Buffer board 


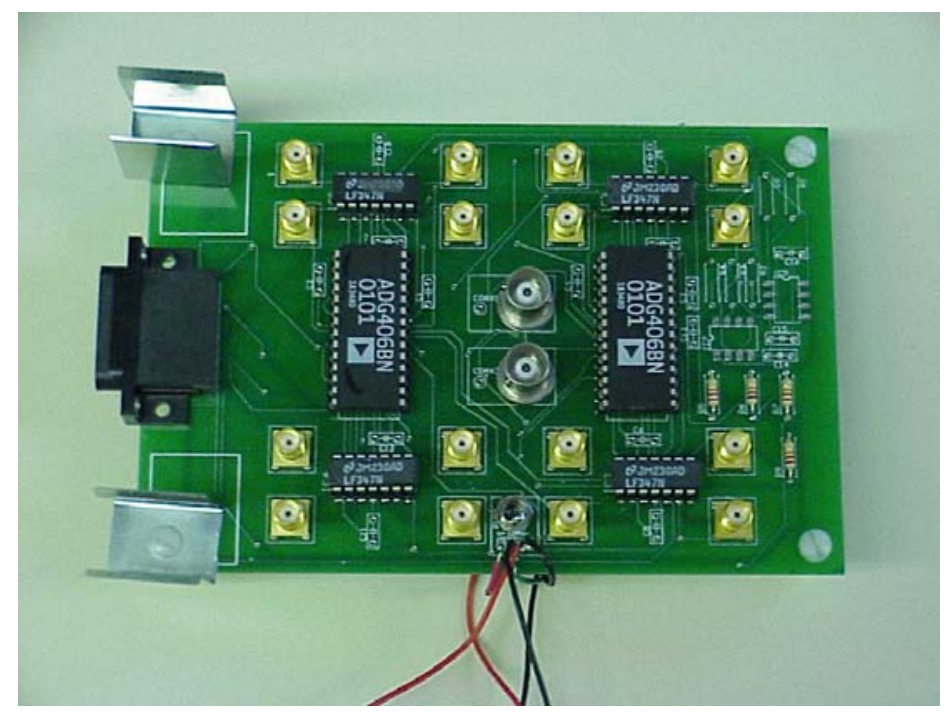

Fig. 13. Multiplexer and buffering board

A new 64-element sensor board has superseded the 16-1 buffering and multiplexing board shown in Figure 13. The new 64-element sensor board has incorporated several design enhancements, such as the implementation of the buffering and multiplexing functionality directly on the topside of the sensor board. The 64-element board uses surface mount quad op amps and multiplexers that mount directly to the top surface of the sensor to reduce the size and complexity of the support electronics.

The 64-element sensor divides the array into functional groups of 16 elements with their own local multiplexer and buffering amplifiers. A quad LF412 amplifier is located at the center of every 4 sense-element group. At the center of every 16 sense-element group there is located a 16 to 1 multiplexer to gather the output of the 4 quad amps into one channel. A close-up of such a group is shown in figure 16. The 64-element sensor board uses 16 quad op amps and 4 sixteenchannel multiplexers.

In addition to simplifying the wiring harness, the use of surface mount components should help normalize the sensitivity of the array elements. Some of the variation now seen can be attributed to the cabling between the sense elements and the first stage of amplification. Long cable runs produce a parasitic capacitance in parallel with the sense element. The additional capacitance decreases the sensitivity in a less than predictable fashion. Additionally it is anticipated that this normalized sensitivity will allow the sensor design to replace the variable tuning capacitors with a fixed capacitance drive configuration. 


\section{$\underline{\text { Sensor Development }}$}

Experience gained from the latest driven sensor array has allowed for the completion of the 64-element sensor array. This increase in the number of sense elements will correspondingly increase the resolution of the image generated. This array is composed of 64, 2"x 2 " sensor elements embedded in a grounded mesh. The backside of the sensor array has a RF drive signal distribution array.

The 64-element sensor array is composed of four layers. The first layer or bottom of the board is composed of the 64 sense elements and the mesh that surrounds the sense elements. This layer is shown in Figure 14. Each of these elements is approximately 2" x 2". The next layer up from the bottom sense layer is the RF drive layer, this layer routs the $100 \mathrm{kHz}$ input or drive signal to the 64 elements via the variable tuning capacitors. The next layer up from the drive layer is the shielding layer this layer is tied to the bridge ground. It is expected that a greater uniformity of electric field line distribution will also result from the higher element count. This field uniformity should translate into a flatter response from one element to the next for a given signal. This results from the increase in the number of elements that do not lie on the edge of the array.

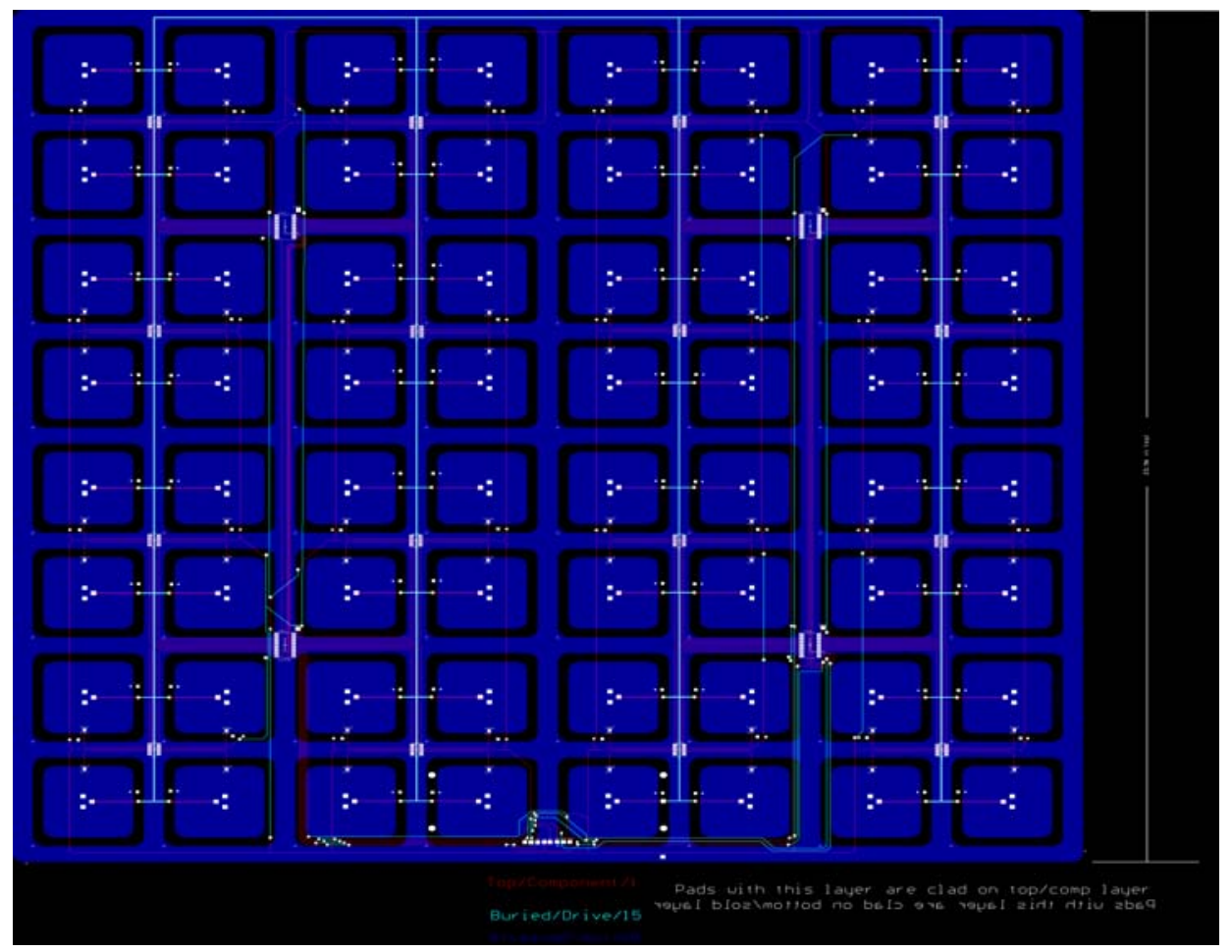

Fig. 14. 64-Element Sensor Board Layout 


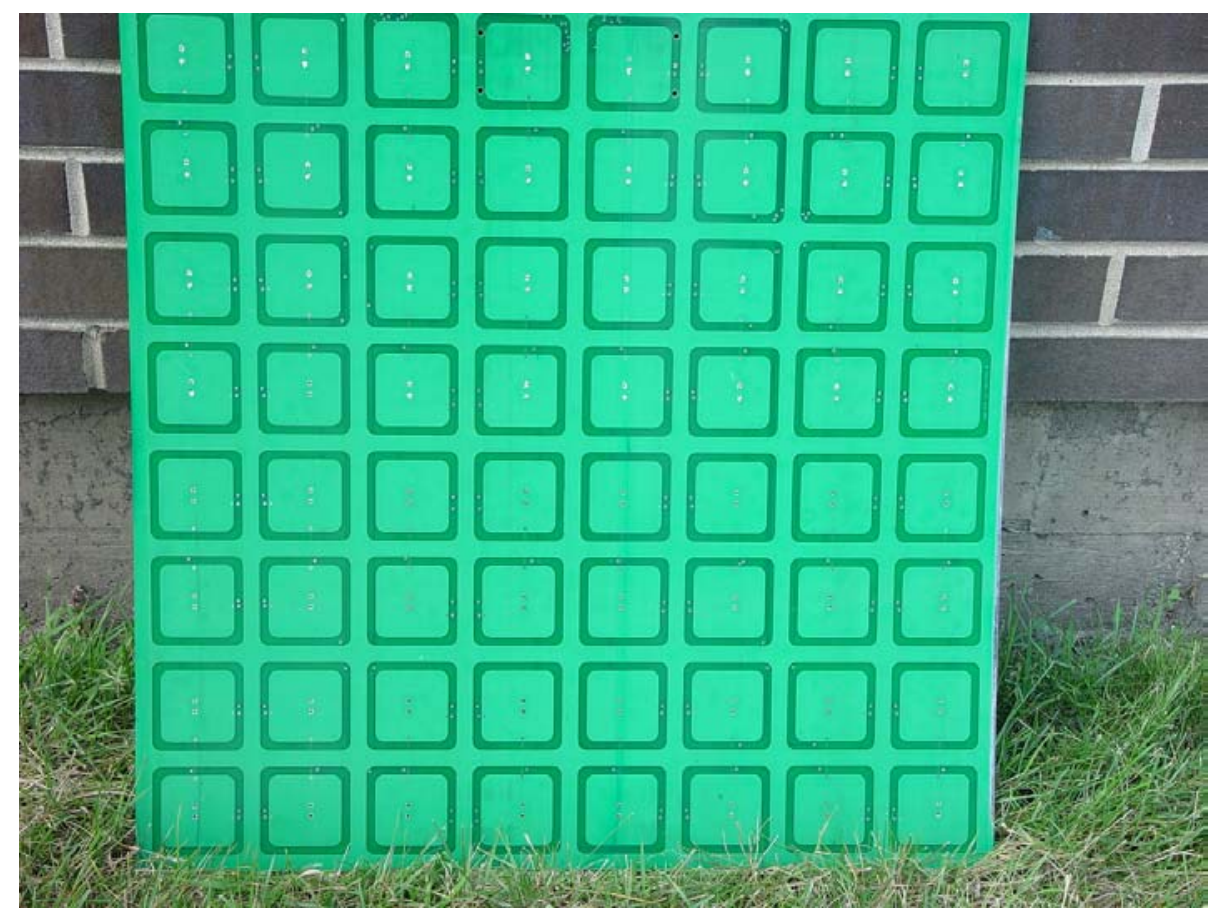

Fig. 15. Sensor Side of Completed 64-Element Board

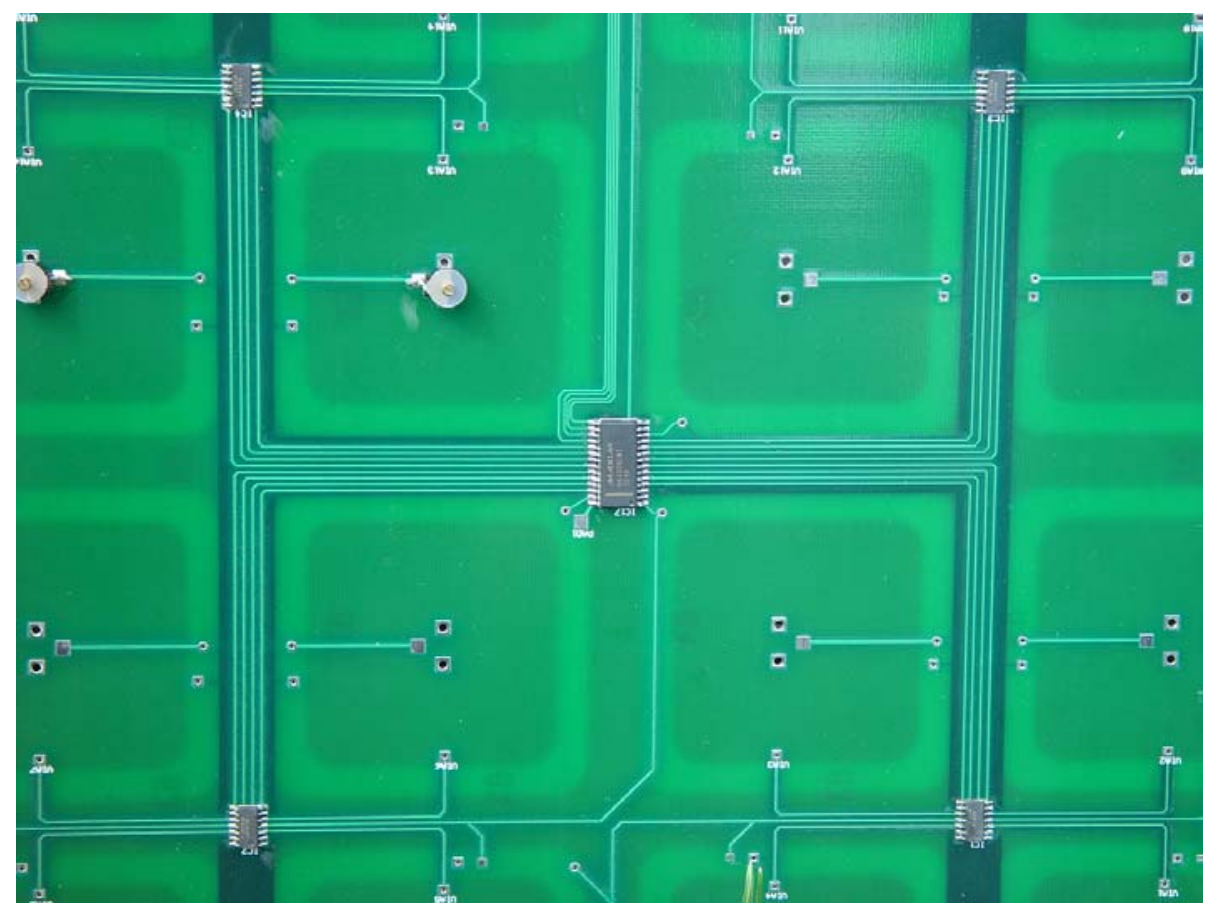

Fig. 16. Close Up of Component Side Showing Multiplexer and Amplifiers 


\section{$\underline{\text { Sensor Array Mounted to Cart }}$}

In order to facilitate the practical imaging of a plastic pipe buried in the field, the 16element sensor array was mounted to a wheeled cart. This cart is constructed of wood and has an aluminum handle that allows the user to easily lift the array off the surface move it to the area of interest and set it down. The array rests on a 1/8-inch thick sheet of anti static polycarbonate mounted to the bottom of the cart. The cart was constructed so that on perfectly flat ground there would be an air gap of $1 / 8$ of an inch. Obviously in most field test situations the ground is not perfectly flat and it is expected that the array will be in to some large degree in contact with the ground surface. Testing of the cart array system is in the early stages and no data was available at the time of this writing.

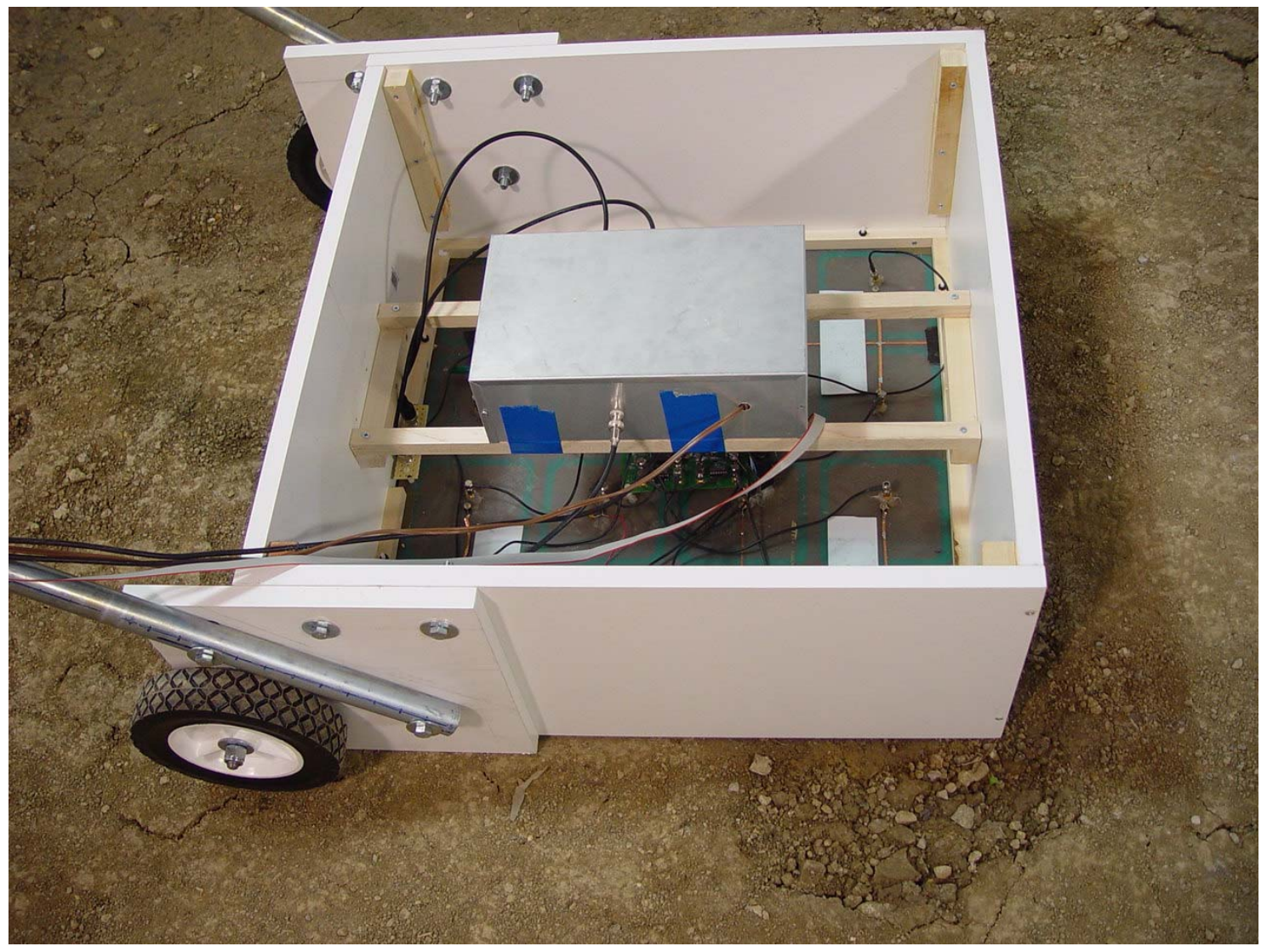

Fig. 17. Cart with 16-Element Sensor array and Electronics 


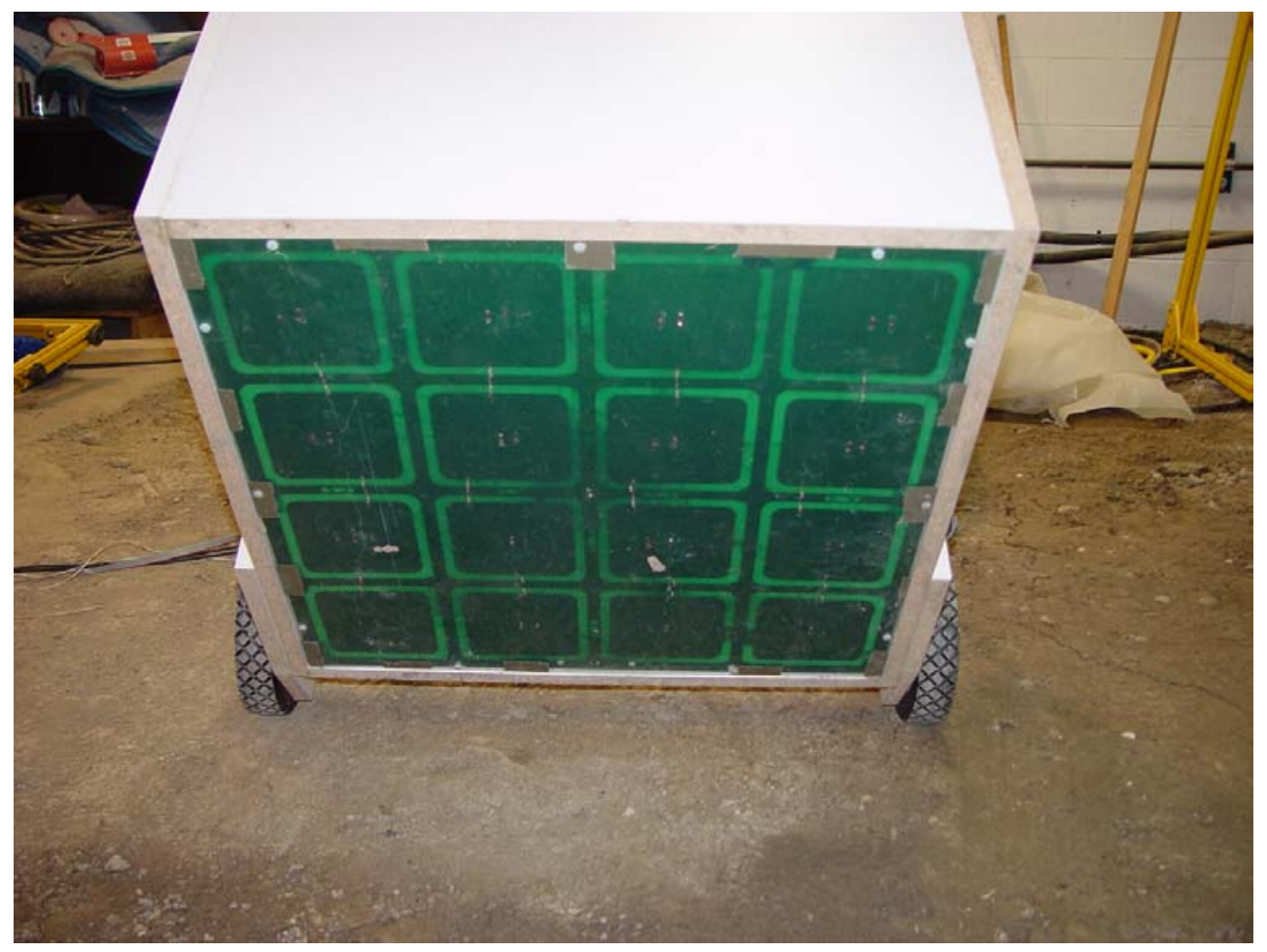

Fig. 18. Bottom of Cart Showing Array

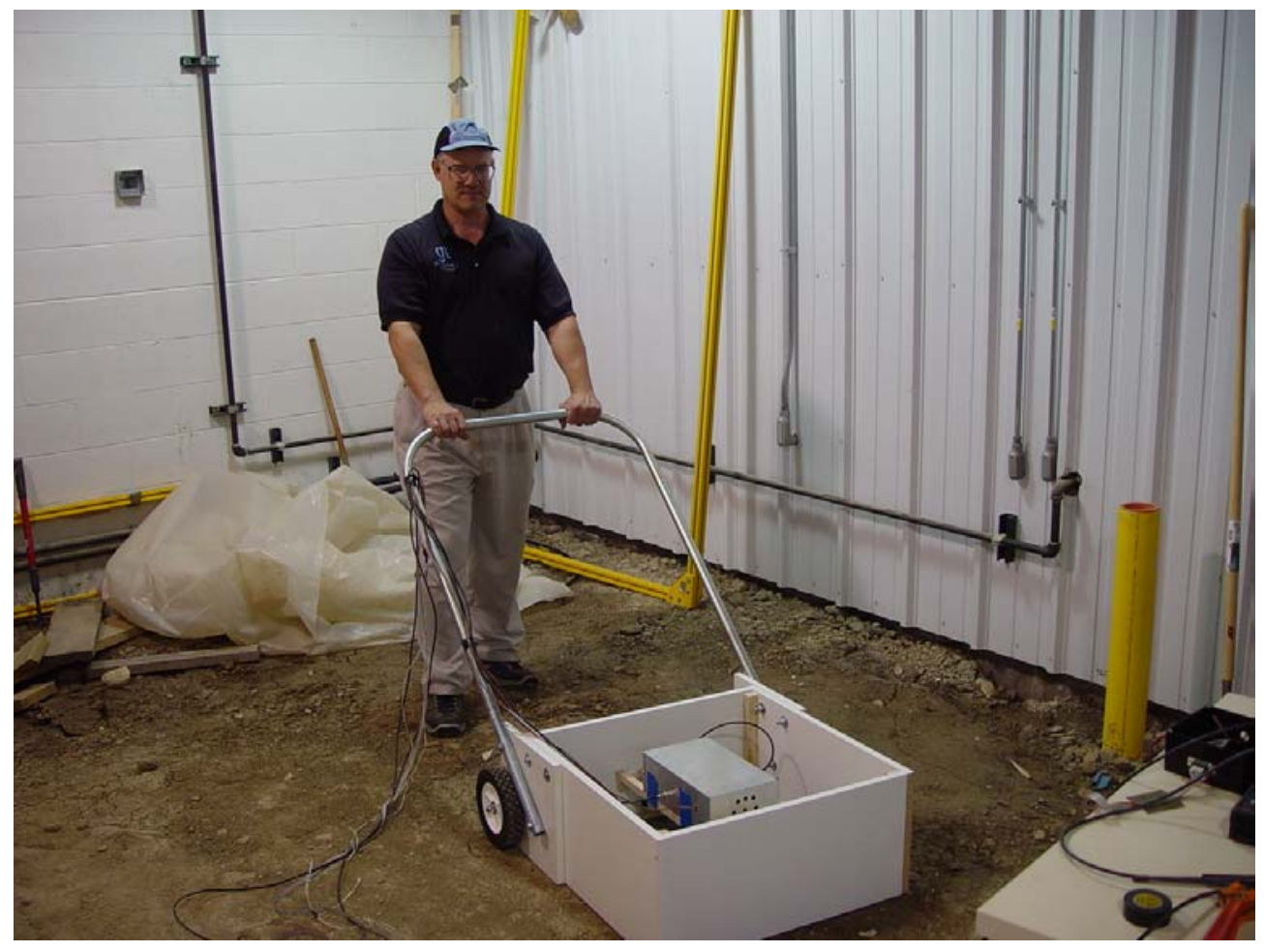

Fig. 19. Cart and Operator 


\section{CONCLUSIONS}

- The 16-element array has sufficient sensitivity to detect PE pipe in wet soils at depths greater than 4 feet.

- The technique uses a combination of phase detection and signal averaging to achieve the required sensitivity.

- The signal to noise ratio is sufficient that a 64-element array was designed and fabricated with the full expectation of achieving better image resolution.

- There are still issues with the achieving uniform sensitivity with every sense element in the array.

- The 64-element sensor array will make use of surface mount components and printed circuit techniques to normalize the sensitivity. 


\section{Work Performed in the second Quarter of 2003}

\section{$\underline{\text { Task 1: Research Management Plan }}$}

Four Quarterly Technical Reports covering the period from October of 2001 through March of 2003 have been submitted. Dan Driscoll of NETL visited GTI in May of 2002 and was briefed on the progress in the project and given a tour of the facilities. The NETL project manager has been provided with periodic brief updates. The progress on the CT project was presented at the "Natural Gas Infrastructure Reliability Forum" in Morgantown on September $17^{\text {th }}$. A paper was also presented at the Natural Gas Technologies Conference in Orlando on October $1^{\text {st }}$.

\section{Task 2: Fabrication of the Sensor Array}

A new 64-element sensor board was fabricated and tested. This board improves the image resolution and integrates the sensor electronics into the sensor board, removing the need for a separate board to be mounted to the sensor array. Testing and integration of the board was completed.

\section{Task 3: Design and Prototype Support Electronics}

Solid State bridge amplifier was mounted in an aluminum box with a power supply and cooling fan. The new sensor board incorporates many of the necessary components as surface mount packages directly on the back of the sensor board. This greatly reduces the bulk and complexity of the wiring harness.

\section{Task 4: Construct Field-Ready Mat Prototype}

Lessons learned from the new 16-element sensor board were incorporated into a new 64element sensor board. The design for this board is ready for fabrication. Integrating the drive elements into the sensor board and using SMA connectors have improved the physical robustness of the sensor.

A portable cart was designed and constructed. A 16-element board was mounted to the bottom of this cart. The multiplexer and amplifier electronics was placed and mounted inside the cart. This arrangement enables an operator to easily push the sensor array over the area of interest to take an image. 


\section{Task 5: Demonstrate Mat Prototype}

There is an opportunity for a public demonstration of the CT Array sensor in July of 2003. The Underground Focus Live trade show will be held in Manteno, Illinois on July $23^{\text {rd }}$ and $24^{\text {th }}$. This show will have exhibitors from many facets of the underground construction industry. GTI plans to show the CT Array prototype at UFL in order to place it before potential manufacturers and end-users.

At this writing GTI has reserved booth space and begun the necessary arrangements to participate in this show.

\section{$\underline{\text { Technical Problems Encountered }}$}

No technical problems that will impact the ability to perform the project or project schedule have been encountered.

\section{$\underline{\text { Project Management Problems Encountered }}$}

The project schedule has been extended through August of 2003 as requested. This is a no cost time extension. The extension should allow significant outdoor testing of the sensor system to be carried out.

\section{Work Planned For The 3rd Quarter Of 2003}

- Testing of hardware and software on GTI's plastic pipe test bed

- Improve and enhance horizontal and vertical imaging of sub-surface plastic pipe.

- Continue to enhance the sensitivity of the sensor array.

- Test and integrate 64-element array into a functioning subsurface imaging system.

- Simplify the imaging used in the operator interface. 


\section{REFERENCES}

In a patent entitled " Driven Shielding Capacitive Proximity Sensor", patent number 5,166,679, dated November 24, 1992, inventors John M. Vranish and Robert L. McConnell have presented an invention for a capacitive proximity sensor that will detect the intrusion of a foreign object into the working space of an electrically grounded robotic arm. The capacitive proximitysensing element is backed by a reflector that is driven by an electrical signal of the same amplitude and phase as that signal which is detected by the sensor. It is claimed that by driving the reflector plate with the same signal that is on the sense element significant increases in the sensor's range and sensitivity are accomplished.

In a patent entitled "Steering Capaciflector Sensor", patent number 5,363,051, dated November 8, 1994, inventors Del T. Jenstrom and Robert L. McConnell, present an invention that will allow for the steering of the electric field lines produced by a capacitive type proximity sensor. The inventors assert the claim that by steering or focusing the electric field will allow an increased ability to discriminate and determine the range of an object in the area of observation over that of previous capacitive sensors. Differential voltages applied to shielding plates spatially arranged around the sensor plate accomplish steering of the electric field lines.

In a patent entitled "Buried Pipe Locator Utilizing A Change In Ground Capacitance", patent number 5,617,031 dated April 1, 1997 inventor John E. B. Tuttle has invented a portable buried pipe detection device that utilizes changes in the electrical properties of the soils surrounding underground pipes. The detection method consists of the injection of a low frequency sinusoidal wave into the ground via an array of injector/sensor plates. Subsequent modification of the injected signal by variations in ground impedance brought about by the existence of buried piping structures will result. The modified signals will be detected by the spatially separated sensor elements located on the device. The injector/sensor elements are constructed in such a manner as to comprise a capacitive bridge circuit when viewed in conjunction with the ground. As the detection array is moved along the ground any occurrence of underground piping structures will imbalance the capacitive bridge and give rise to a detectable electrical signal. 


\section{LIST OF ACRONYMS AND ABBREVIATIONS}

CT - Capacitive Tomography

COR - Contracting Officer's Technical Representative

DOE - Department of Energy

FERC - Federal Energy Regulatory Commission

GPR - Ground Penetrating Radar

GRI - Gas Research Institute

GTI - Gas Technology Institute

IGT - Institute of Gas Technology

IRNG -Infrastructure Reliability of Natural Gas

PCB - Printed Circuit Board

IF - Intermediate Frequency

MDPE - Medium Density Polyethylene

VI - Virtual Instrument

NI - National Instruments

DACQ - Data Acquisition

GUI - Graphical User Interface

SNR - Signal to Noise Ratio 


\section{Appendix A. MatLab Code for Sensor Analyses}

$\%$ This function calculates various impedence parameters for two resistors $\%$ and capacitors in series. More importantly it calculates the variation in $\%$ voltage across the second impedance(Z2)due to a small variation in the \%capacitance of Z2. This is given by the input variable sm_del, which takes $\%$ on values $0<\mathrm{sm}$ del $<1$. The basic equation is $\mathrm{V} 2=[\mathrm{A}][\mathrm{B}]$ and $\% \mathrm{~V} 2$ del $=\left[\mathrm{A} \_\right.$del $][\mathrm{B}$ _del], $\mathrm{A}$ is the impedance due to the pure magnitude of the \%parameters, A_del is the same except calculated with $\mathrm{C} 2$ having changed a $\%$ small amount. B is the voltage variation due to the phase factor and \%likewise B_del is that due to the addition of the variation in C2. At the $\%$ end a subtraction is done to compare the voltage variation due to the $\%$ change in magnitude and the change in phase. Assuming the code is correct $\%$ where the reactance is greater than the resistance the phase factor $\%$ predominates the reverse is true where the resistance is greater than the $\%$ reactance. The drive voltage has been assumed to be 1 volt. Multiplying the $\%$ Z_Mag_Change or Z_Phs_Change by the actual drive voltage gives the true \%ouput variation. The effect of the phase factor can not be seen if one is $\%$ averaging over many cycles, if for instance one is taking one sample per $\%$ cycle the phase effect is observable.

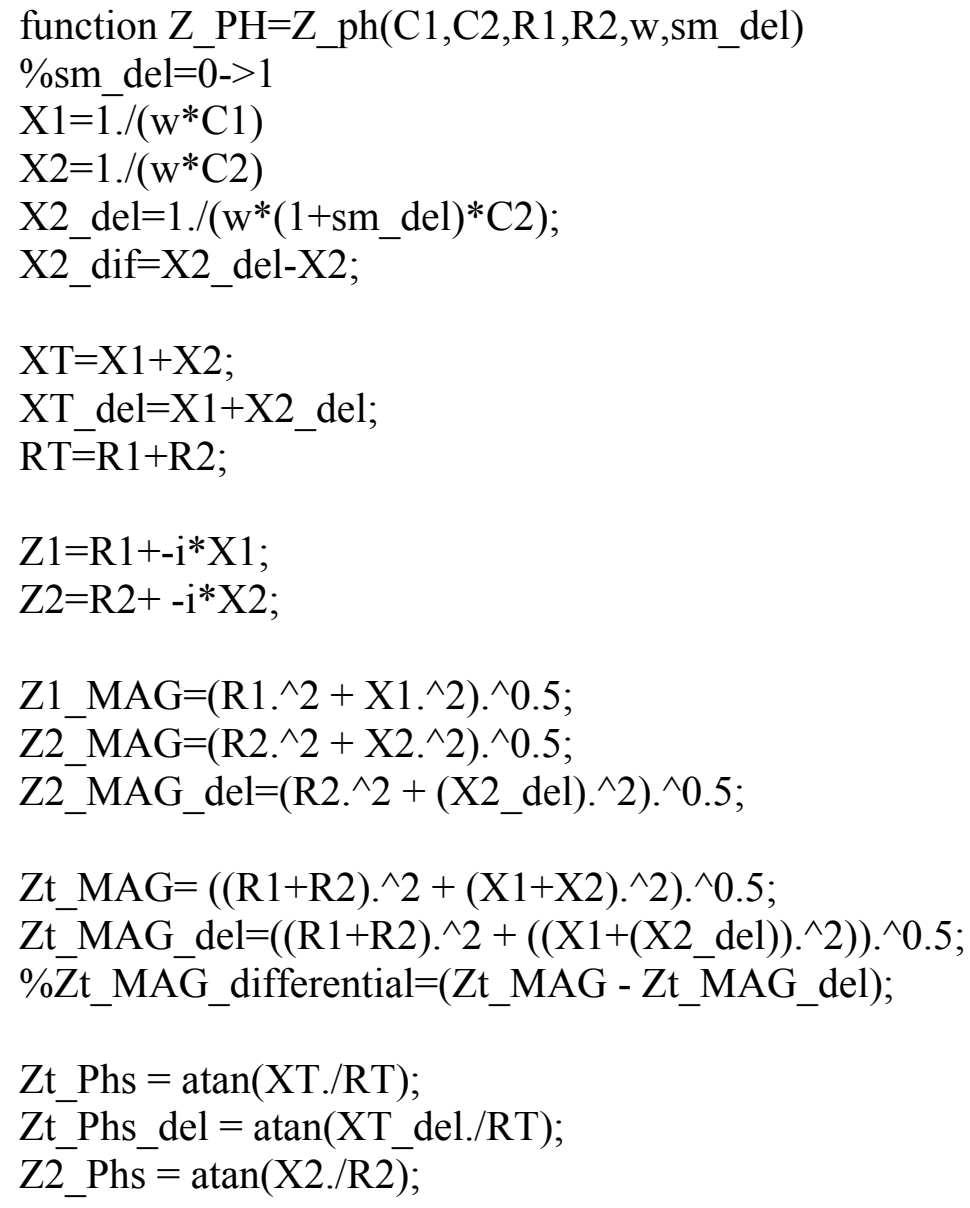


Z2_Phs_del = atan(X2_del./R2);

V2_Phs_sens $=(\mathrm{Z} 2$ Phs $-\mathrm{Zt}$ t Phs $)$;

V2_Phs_sens_del=(Z2_Phs_del - Zt_Phs_del $)$;

$\mathrm{A}=\left(\mathrm{Z} 2 \_\right.$MAG./Zt_MAG);

A_del=(Z2_MAG_del./Zt_MAG_del);

Z_Mag_Change $=\bar{A}-A \_$del

$\mathrm{B}=\sin \left(\mathrm{V} 2 \_\mathrm{Phs} \_\right.$sens $)$;

$\mathrm{B}$ del $=\sin (\mathrm{V} 2$ Phs sens del);

$\mathrm{Z}$ Phs Change $=\bar{B}-\mathrm{B}$ del

$\% \mathrm{~V}$ sensor $=(\mathrm{A}) *(\mathrm{~B})$

$\% \mathrm{~A}=\mathrm{Z} 2$ del./(Zt_MAG_del)

$\% \mathrm{~B}=\mathrm{ph} \_$Tot

Z_PH=Zt_MAG;

function $Z_{\text {deriv }}=\mathrm{z}$ der $(\mathrm{z} 1, \mathrm{z} 2)$;

$\mathrm{Z}$ deriv $=(\mathrm{z} 1 * \mathrm{z} 2+\mathrm{z} 2 . \wedge 2) . /(\mathrm{z} 1+\mathrm{z} 2) \cdot{ }^{\wedge} 3$;

MatLab Code for Calculating the Derivative and Creating the above Plot. 